\title{
A systematic review and meta-analysis of enrollment into ARDS and sepsis trials published between 2009 and 2019 in major journals
}

Dustin C. Krutsinger ${ }^{1 *}\left(\mathbb{D}\right.$, Kuldeep N. Yadav ${ }^{2,3}$, Michael O. Harhay ${ }^{2,3,4}$, Karsten Bartels $^{5}$ and Katherine R. Courtright $2,3,4$

\begin{abstract}
Background: Enrollment problems are common among randomized controlled trials conducted in the ICU. However, little is known about actual trial enrollment rates and influential factors. We set out to determine the overall enrollment rate in recent randomized controlled trials (RCTs) of patients with acute respiratory distress syndrome (ARDS), acute lung injury (ALI), or sepsis, and which factors influenced enrollment rate.
\end{abstract}

Methods: We conducted a systematic review by searching Pubmed using predefined terms for ARDS/ALI and sepsis to identify individually RCTs published among the seven highest impact general medicine and seven highest impact critical care journals between 2009 and 2019. Cluster randomized trials were excluded. Data were extracted by two independent reviewers using an electronic database management system. We conducted a random-effects metaanalysis of the eligible trials for the primary outcome of enrollment rate by time and site.

Results: Out of 457 articles identified, 94 trials met inclusion criteria. Trials most commonly evaluated pharmaceutical interventions (53\%), were non-industry funded (78\%), and required prospective informed consent (81\%). The overall mean enrollment rate was 0.83 (95\% confidence interval: $0.57-1.21$ ) participants per month per site. Enrollment in ARDS/ALI and sepsis trials were $0.48(95 \% \mathrm{Cl} 0.32-0.70)$ and $0.98(95 \% \mathrm{Cl} 0.62-1.56)$ respectively. The enrollment rate was significantly higher for single-center trials $(4.86 ; 95 \% \mathrm{Cl} 2.49-9.51)$ than multicenter trials $(0.52 ; 95 \% \mathrm{Cl} 0.41-0.66)$. Of the 36 trials that enrolled $<95 \%$ of the target sample size, 8 (22\%) reported slow enrollment as the reason.

Conclusions: In this systematic review and meta-analysis, recent ARDS/ALI and sepsis clinical trials had an overall enrollment rate of less than 1 participant per site per month. Novel approaches to improve critical care trial enrollment efficiency are needed to facilitate the translation of best evidence into practice.

Keywords: ARDS, Sepsis, Acute lung injury, Randomized controlled trial, Enrollment

\footnotetext{
*Correspondence: Dustin.Krutsinger@unmc.edu

1 Division of Pulmonary, Critical Care, and Sleep Medicine, University of Nebraska Medical Center, 985910 NE Medical Center, Omaha, NE 68198, USA

Full list of author information is available at the end of the article
}

\section{Background}

Enrollment problems in randomized controlled trials (RCTs) lead to reduced precision regarding interventions' effects [1], increased study costs [2], and slows the adoption of evidence into practice [3]. Research involving critically ill patients poses unique challenges to trial enrollment, including narrow recruitment windows and 
reliance on surrogate decision-makers for obtaining informed consent $[4,5]$. Moreover, critical care trials are often underpowered to detect a clinically meaningful difference in mortality due to, at least in part, enrollment difficulties [6] Additionally, several recently published high profile critical care trials were terminated early due to difficulties with enrollment [7-9].

Underpowered trials, and those terminated strictly due to feasibility issues, raise ethical concerns as they expose participants to any risks involved in participation with a reduced likelihood of social benefit [10]. In an effort to address issues of trial inefficiencies, it has been proposed that alternative recruitment strategies [11] and trial designs [12] are needed. Accurate estimation of enrollment rate is essential to inform trial planning and to serve as a benchmark for evaluating the utility of novel trial designs. However, reporting of trial recruitment and enrollment metrics varies, and little is known about trial factors that influence the enrollment rate.

We conducted a systematic review of trials that evaluated interventions for common critical care syndromes, including acute respiratory distress syndrome (ARDS), acute lung injury (ALI), and sepsis, to assess enrollment rate and contributing trial design factors. We focused this review on trials published in the last decade to ensure the results reflect contemporary critical care science and recruitment practices to best inform trial design and conduct in the near future.

\section{Methods}

We conducted a systematic review and meta-analysis, following the Preferred Reporting Items for Systematic Reviews and Meta-Analyses guidelines (Additional file 1: Appendix A) [13]. The protocol was not eligible for registration in the International Prospective Register of Systematic Reviews since the outcomes of interest were not health-related [14]. This study did not meet the criteria for human subjects research as defined by United States federal policy 45 Code of Federal Regulations 46 and thus was exempt from review by the institutional review board.

\section{Data sources}

We searched PubMed to identify all ARDS/ALI and sepsis RCTs published from 2009 to 2019 in the seven highest-impact critical care and seven highest impact general medicine journals according to InCites Journal Citation Reports [15]. The following journals met our criteria: (1) The New England Journal of Medicine, (2) Lancet, (3) Journal of the American Medical Association, (4) Nature Reviews Disease Primers, (5) British Medical Journal, (6) JAMA Internal Medicine, (7) Annals of Internal Medicine, (8) Lancet Respiratory Medicine, (9) Intensive Care
Medicine, (10) American Journal of Respiratory and Critical Care Medicine, (11) Chest, (12) Critical Care, (13) Critical Care Medicine, and (14) Annals of the American Thoracic Society. Consistent with prior systematic reviews that limited the literature search to high-impact journals when asking questions related to trial design and metrics $[6,16]$, we restricted our search to high-impact journals in order to increase the likelihood of capturing the highest quality trials and thus are most likely to reflect the "best-case scenario" of enrollment rate. We used the following Medical Subject Headings (MeSH) terms: (1) respiratory distress syndrome, adult, (2) sepsis, and (3) acute lung injury. The full search strategy was determined a priori and is detailed in Additional file 1: Appendix B.

\section{Study selection and data extraction}

Two reviewers (D.C.K., K.N.Y) independently screened abstracts and full-text articles for pre-specified eligibility criteria. Articles were included for full-text review if they were conducted among patients diagnosed with ARDS/ ALI or sepsis in the intensive care unit or emergency department, randomized at the patient level, required informed consent, and reported at least one clinical outcome. The risk of bias for trial outcomes was not assessed for this review of trial process metrics.

\section{Outcomes}

The primary outcome was enrollment rate, defined as the number of participants enrolled in each trial per month per site. The secondary outcome was the reported reason that target enrollment (randomized $<95 \%$ of target sample size) was not achieved.

\section{Data synthesis and analysis}

Two reviewers (D.C.K., K.N.Y) independently extracted the data from full-text articles using REDCap electronic database [17]. The primary outcome of enrollment rate was calculated by dividing the number of participants enrolled in each trial by the duration of the trial (in months) and then by site. Duration of enrollment was determined from the reported start, and end dates rounded to the nearest month. Trials that did not report a start and stop date for enrollment or did not report the number of participating study sites were not included in the meta-analysis. Study heterogeneity was assessed using Cochran's $Q$ and the $I^{2}$ statistic [18]. Due to the significant heterogeneity across studies for the primary outcome, we performed a random-effects meta-analysis to determine the overall enrollment rate using the method of DerSimonian and Laird [19], which adjusts the standard errors of the study estimates to account for studyspecific heterogeneity such as sample size. 
We conducted a stratified analysis of the primary outcome, stratified by condition ARDS/ALI and sepsis, excluding trials that combined conditions. We conducted the following pre-specified subgroup analyses for the primary outcome: (1) the number of sites (single-center versus multicenter), (2) temporal trend (publication year 2009-2013 versus 2014-2019), (3) funding source (any versus no industry funding), (4) intervention (pharmaceutical versus non-pharmaceutical), (5) continent (first author's location), (6) informed consent (prospective versus retrospective), and (7) enrollment achieved ( $\geq 95 \%$ versus $<95 \%$ of target sample size randomized).

All statistical analyses were performed using the $R$ language for statistical computing (version 4.0.1), and $\mathrm{R}$ packages meta (version 4.13-0) and tidyverse (version 1.3.0).

\section{Results}

\section{Characteristics of included studies}

Our PubMed search yielded 457 unique articles, of which 94 met eligibility criteria (Fig. 1, Additional file 1: Appendix C). Most trials evaluated pharmaceutical interventions (53\%), were non-industry funded (78\%), and required prospective informed consent (81\%) (Table 1). Recruitment occurred at a median of 18 (IQR 2-38) sites per trial for a median duration of 36 (IQR 24-48) months (Additional file 1: Appendix D).

\section{Enrollment rate}

Two trials did not report their enrollment duration and were excluded from the primary analysis. The overall mean enrollment rate was 0.83 (95\% confidence interval [CI] 0.57-1.21) participants per month per site (Fig. 2). ARDS trials had an enrollment rate of 0.48 (95\% CI 0.320.70 ), while sepsis trials had an enrollment rate of 0.98 (95\% CI 0.62-1.56). Study heterogeneity was assessed using a funnel plot (Fig. 3).

The enrollment rate was significantly higher in singlecenter (4.86; 95\% CI 2.49-9.51) compared to multicenter trials $(0.52$; $95 \%$ CI $0.41-0.66 ; p<0.0001)$ and in nonindustry funded trials $(0.93$; $95 \%$ CI $0.57-1.52)$ compared to those with any industry funding $(0.42 ; 95 \% \mathrm{CI}$ $0.27-0.64 ; p=0.01$ ). There was a significant difference in enrollment rates by the continent of the first author: Europe 0.57 (95\% CI 0.44-0.75), North America 0.82 (95\% CI 0.39-1.74), Australia 0.95 (95\% CI 0.55-1.62), South America 1.85 (95\% CI 0.81-4.22) and Asia 1.89 (95\% CI $0.72-4.97 ; p=0.01$ for overall comparison). Trials with the first author from Asia (47\%) and South America (38\%) were more likely to be single center trials than those originating from North America (19\%) and Europe (13\%) (Additional file 1: Appendix E).

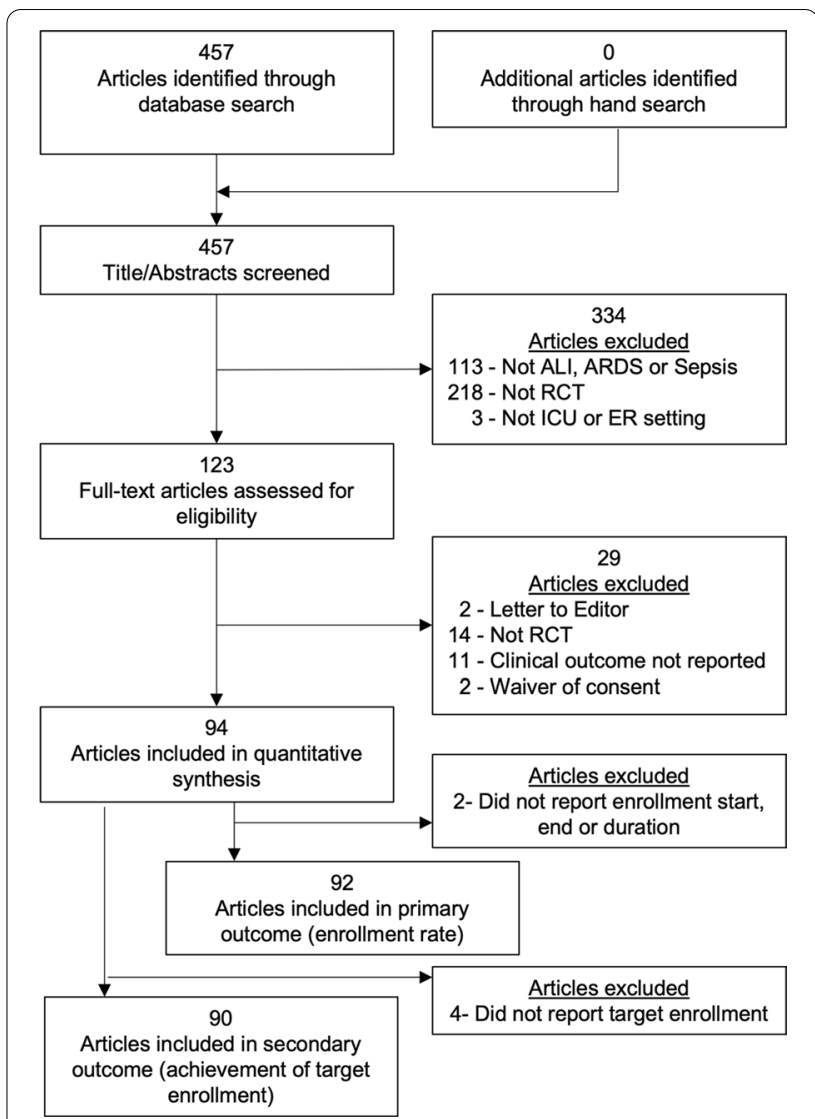

Fig. 1 Article selection through the systematic process

Enrollment rates did not differ by publication year (2009-2013: 0.62; 95\% CI 0.41-0.92 vs 2014-2019: 1.08; 95\% CI $0.63-1.85 ; p=0.10$ ), intervention (pharmaceutical: 0.81 ; $95 \%$ CI $0.44-1.50$ vs non-pharmaceutical: 0.85 ; 95\% CI $0.56-1.31 ; p=0.90$ ), or method of informed consent (retrospective permitted: 0.65 ; $95 \%$ CI $0.48-0.89$ vs prospective required: $0.89 ; 95 \%$ CI $0.59-1.35 ; p=0.25$ ). Detailed article-level and summary results by study characteristics are available in Additional file 1: Appendices F through P.

\section{Achievement of target enrollment}

Four trials did not report a target sample size. Of the 90 trials reporting a target sample size, 36 trials (40\%) failed to enroll at least $95 \%$ of the target sample size. The most commonly cited reasons for trials falling short of their enrollment target included intervention futility $(n=15$; $42 \%)$, safety concern $(n=10 ; 28 \%)$, and slow enrollment $(n=8 ; 22 \%)$. Characteristics of trials that cited slow enrollment for falling short of their enrollment target are included in Additional file 1: Appendix Q. Enrollment rates did not differ between trials that failed to reach $95 \%$ of the targeted sample size $(0.59 ; 95 \%$ CI $0.38-0.91)$ 
Table 1 Characteristics of articles

\section{Characteristic}

Included studies

Target patient population

Acute respiratory distress syndrome (ARDS)/acute lung injury (ALI)

Sepsis (includes sepsis, severe sepsis, septic shock)

Both sepsis and ARDS

Publication period

2009-2013

2014-2019

Journal

American Journal of Respiratory and Critical Care Medicine

Chest

Critical Care

Critical Care Medicine

Intensive Care Medicine

Journal of the American Medical Association

Journal of the American Medical Association - Internal Medicine

Lancet

Lancet-Respiratory Medicine

New England Journal of Medicine

Continent of first author

Asia

Australia

Europe

North America

South America

Funding sources $^{a}$

Academic institution

Association or foundation

Government

Industry

Unreported

Intervention ${ }^{b}$

Clinical protocol

Device

Diagnostic test

Drug

Consent type

Prospective

Retrospective

Enrollment achieved ${ }^{c}(N=90)$

$\geq 95 \%$ of target sample size enrolled

$<95 \%$ of target sample size enrolled

Futility $^{a}$

Safety $^{\text {a }}$

Efficacy ${ }^{\text {a }}$

Slow enrollment ${ }^{\mathrm{a}}$

Other/not reported ${ }^{\mathrm{a}}$
Number

Percent

94

23

${ }^{a}$ Not mutually exclusive, ${ }^{b}$ One article reported both a pharmacotherapeutic and clinical protocol intervention, ${ }^{\mathrm{c}}$ Four trials did not report a sample size target 


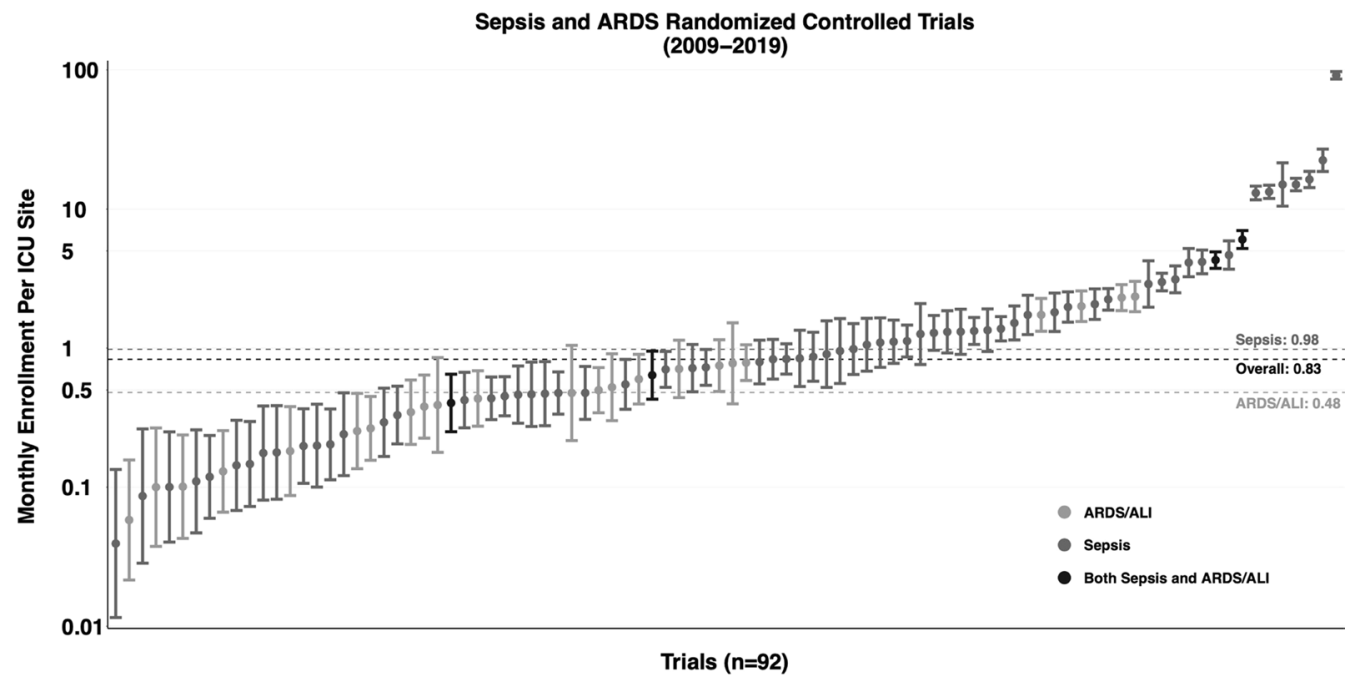

Fig. 2 Enrollment rate among ARDS/ALI and sepsis trials published 2009-2019. Enrollment rate defined as the number of participants enrolled per month per site, displayed on log scale. Two trials excluded due to failure to report enrollment duration

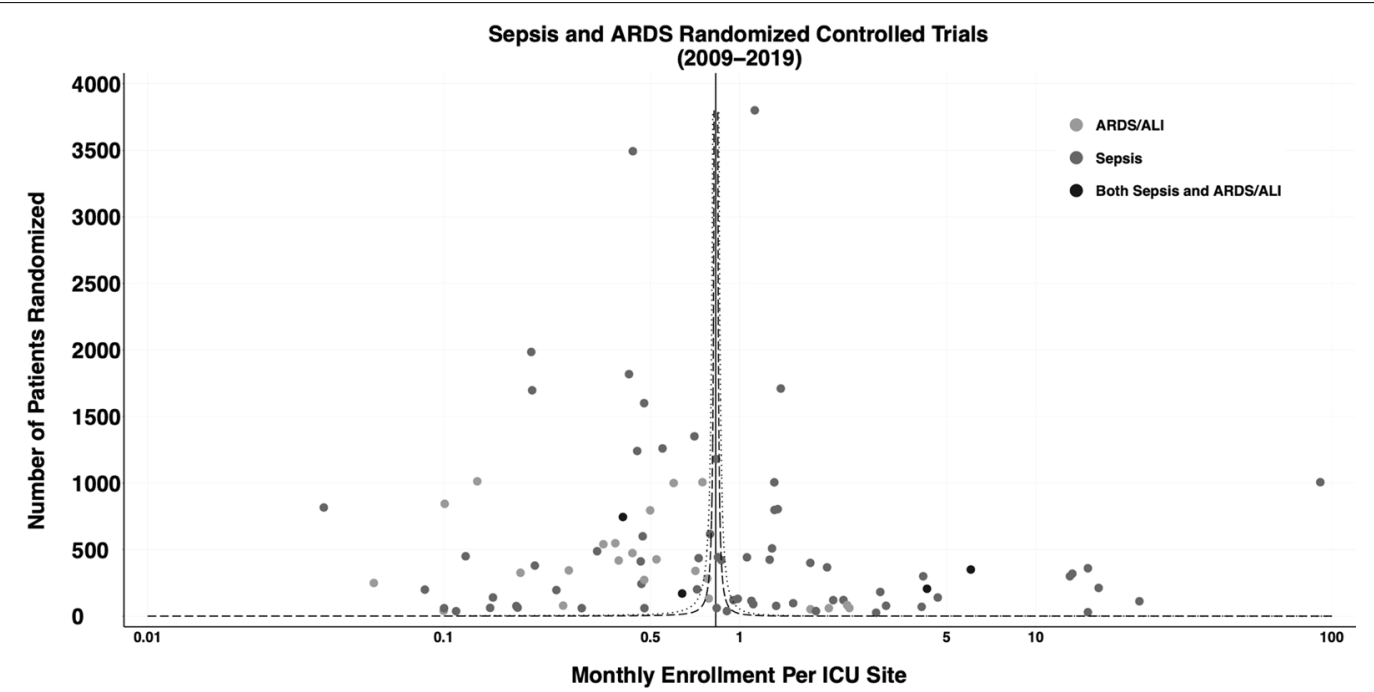

Fig. 3 Funnel plot. Number of patients randomized against enrollment rate per month per site, displayed on a log scale. The vertical dotted line represents the overall monthly enrollment rate per site. The two exponential lines represent the 95\% (grey, small dashed line) and $99.8 \%$ (black, large dashed line) confidence intervals around the overall monthly enrollment rate per site

compared to trials that met or exceeded $95 \%$ of the targeted sample size $(1.03 ; 95 \%$ CI $0.62-1.72 ; p=0.10)$.

\section{Discussion}

In this systematic review of 94 contemporary ARDS/ALI and sepsis trials, we found an overall enrollment rate of less than 1 participant per month per site, and almost 1 in 10 trials failed to meet the target sample size due to slow enrollment. These findings have important implications for trial planning and provide evidence to support the general perception among critical care trialists that conducting trials among critically ill patients is arduous, and novel interventions to improve enrollment, trial efficiencies, and alternative designs are needed [12].

Our overall findings are consistent with a previous study that found a median enrollment rate of 0.90 patients per site per month among 23 multicenter RCTs of non-surgical interventions for adult critically ill patients [20]. Another prior review found that $\geq 95 \%$ of target enrollment was not achieved in $15 \%$ of adult 
critical care trials due to recruitment or logistical issues [6]. Although this proportion was lower in our focused review of ALI/ARDS and sepsis trials, both are likely to be underestimates since trials that fail to achieve enrollment targets may be more likely to remain unpublished or be published in lower-impact journals, which were not included in either review. Furthermore, studies that are underpowered or stopped early due to enrollment difficulties expose enrolled participants to potential risk with reduced likelihood of benefit, thus raising ethical concerns in terms of not providing societal benefit [10].

The overall enrollment rate among sepsis trials was nearly twice that in ARDS/ALI trials, which is likely reflective of important clinical differences between these syndromes that may impact research recruitment processes. For example, patients with ARDS/ALI are likely to be mechanically ventilated and therefore reliant on surrogate decision-makers for research participation decisions. Surrogates often feel overwhelmed by the acuity of the patient's illness and substituted judgment process, which influences their decision-making processes for research participation [21]. Use of ethical and effective behavioral economic nudges during recruitment may help to minimize the surrogate decision-making process and promote informed trial participation [22, 23].

We found several trial-related factors that were associated with better enrollment rates that may help to inform future trial planning. Single-center and non-industry funded trials had higher enrollment rates, which may indicate potential participants' greater trust and confidence in the medical and research teams, important factors in surrogate decision-makers' research participation decisions [24]. Single-center trials may recruit more rapidly in part due to increased motivation of recruiters who likely know or may even be the principal investigator (PI) of the trial. In contrast, multi-center trials face a higher burden to provide consistent recruitment oversight and troubleshooting for site PIs who are crucial to recruitment efforts but also likely to have competing demands on their time. Additionally, there may be differences in the motivation of those recruiting based on the funding source. While adding sites may not be the most efficient solution for trials struggling with slow enrollment, this decision must be balanced with the limited generalizability of evidence from single-center trials. Geographic differences in enrollment rates may partly be explained by differences in the use of single vs multicenter trials and varying requirements for regulatory controls. Interestingly, neither the timing of consent (prospective required versus retrospective permitted), nor type of intervention was associated with enrollment rate in this sample of clinical trials.
Previous qualitative studies have identified factors important to enrollment decisions made by surrogates of critically ill patients. In addition to the importance of trust $[21,25]$, those who elected to enroll the patient cited that they had a desire to help future patients, were following the perceived wish of the patient, had a generally favorable view of research, and perceived the trial as safe. Those who declined enrollment cited the acuity of the patient's illness, a fear of "rocking the boat", and concern with potential interference with the medical team's decisions [4]. The experiences in COVID-19 ICU trial enrollment suggest there are likely additional influential factors on ICU surrogates' decision-making for research participation. For example, the CoDEX trial reported an enrollment rate of 3.6 participants per month per site [26], the HENIVOT trial 13.8 participants per month per site [27], and the INSPIRATION trial 15 participants per month per site [28]. These enrollment rates are significantly higher than what we found in this review of ICU trials conducted before the pandemic, and may be attributable to the allocation of increased resources for recruitment, a feeling of desperation among COVID patients and surrogates motivated to try anything for this unknown and life-threatening disease, or the high volume of COVID patients in the ICU. While research conducted during the COVID-19 pandemic represents an extreme example that is not likely to generalize to most other critical care trials, there may be lessons to glean nonetheless about other important influences on enrollment processes and decisions for participation in critical care research.

Despite multi-center trials being common in this sample, recruitment still required a median of three years to complete. Although the number of eligible patients at each site was inconsistently reported and thus was not included in our analysis, given the ubiquity of ARDS/ALI and sepsis in the ICU [29], it is unlikely that a lack of eligible participants is the primary limiting factor. Indeed, a multicenter study of research recruitment outcomes among critically ill patients found that over half of the opportunities to recruit an eligible patient failed, most commonly due to the research team being unavailable or an inability to contact a surrogate decision-maker [5]. Increasing the number of personnel involved in screening and approaching eligible participants may be an efficient way of increasing the trial enrollment rate, but the increased cost of doing so must also be considered.

Several limitations must be considered when interpreting the results. First, this report only includes trials that enrolled patients with ARDS/ALI or sepsis and were published in select journals. While this intentionally narrow scope may limit the generalizability of the results, we focused on the most common critical illness syndromes and high-impact journals as this is the evidence 
base with the greatest impact on clinical practice. It is likely that trials that experience very slow enrollment or other difficulties achieving their enrollment targets are more likely to be published in lower-impact journals or go unpublished. Therefore, our results likely represent an overestimation of enrollment rates and an underestimation of the problem. Second, due to a lack of reporting of site-level recruitment metrics, our analysis assumes that each site was actively enrolling throughout the duration of the trial. This assumption is likely to be inaccurate for some studies and may have led to an underestimate in the overall point estimates in this review. Third, siteand provider-level factors could play an important role in enrollment, however, important details about such factors are rarely reported in publications. Future research focused on collecting and analyzing site- and providerlevel factors may improve our understanding of factors contributing to successful trial enrollment. Fourth, due to the small numbers in some subgroups, our subgroup analyses may be underpowered. Lastly, we could not determine the overall proportion of eligible participants approached who were enrolled (consent rate) due to significant heterogeneity in reporting. Indeed, adherence to CONSORT reporting guidelines has been notably poor among critical care trials [30], which often experience unique recruitment circumstances (e.g., clinicians declining participation on behalf of the patient or reliance on surrogate decision-makers). Adoption of a standard CONSORT diagram is needed to facilitate an accurate evaluation of consent rates.

\section{Conclusion}

Our review of 94 recent high-impact ARDS/ALI and sepsis trials revealed that enrollment challenges remain an important source of delay in new evidence generation and threaten the scientific validity of trial findings. Novel strategies that surmount modifiable enrollment barriers are urgently needed to improve the efficiency of critical care trials to enable rapid implementation of evidencebased interventions into clinical practice.

\section{Supplementary Information}

The online version contains supplementary material available at https://doi. org/10.1186/s13054-021-03804-1.

Additional file 1. Supplemental information and data. Includes the PRISMA checklist, complete online search strategy, citations for articles included in systematic review and subgroup analyses.

\section{Authors' contributions}

D.C.K, K.N.Y, M.O.H, and K.R.C contributed to the conception and design of the study. D.C.K. and K.N.Y. contributed to the acquisition and analysis of the data. D.C.K, K.N.Y, and K.B. contributed to the interpretation of the data. All authors contributed to the drafting and revising of the manuscript for important intellectual content and approved the final version to be published.

\section{Funding}

Dr. Krutsinger was supported by the National Institute of Health (NIH) (T32 5T32HL098054-09). Dr. Bartels was supported by the NIH, Award Number K23DA040923, and the Agency for Healthcare Research and Quality (AHRQ), Award Number R01HS027795. Dr. Courtright was supported by the NIH (K23HL143181). The content is solely the responsibility of the authors and does not necessarily represent the official views of the $\mathrm{NIH}$ or $\mathrm{AHRQ}$.

\section{Availability of data and materials}

The datasets used during the current study are available from the corresponding author on reasonable request.

\section{Declarations}

Ethics approval and consent to participate

Not applicable.

\section{Consent for publication}

Not applicable.

\section{Competing interests}

The authors declare that they have no competing interests.

\section{Author details}

${ }^{1}$ Division of Pulmonary, Critical Care, and Sleep Medicine, University of Nebraska Medical Center, 985910 NE Medical Center, Omaha, NE 68198, USA. ${ }^{2}$ Palliative and Advanced IIIness Research (PAIR) Center, University of Pennsylvania, 300 Blockley Hall, 423 Guardian Drive, Philadelphia, PA 19104, USA. ${ }^{3}$ Department of Biostatistics, Epidemiology, and Informatics, Perelman School of Medicine, University of Pennsylvania, Philadelphia, PA, USA.

${ }^{4}$ Pulmonary, Allergy, and Critical Care Division, Perelman School of Medicine, University of Pennsylvania, Philadelphia, PA, USA. ${ }^{5}$ Department of Anesthesiology, University of Nebraska Medical Center, 985910 NE Medical Center, Omaha, NE 68198, USA.

Received: 9 August 2021 Accepted: 26 October 2021

Published online: 15 November 2021

References

1. Baldi I, Lanera C, Berchialla P, Gregori D. Early termination of cardiovascular trials as a consequence of poor accrual: analysis of ClinicalTrials.gov 2006-2015. BMJ Open. 2017;7(6):e013482.

2. Emanuel EJ, Schnipper LE, Kamin DY, Levinson J, Lichter AS. The costs of conducting clinical research. J Clin Oncol. 2003;21(22):4145-50.

3. Kahn JM. Disseminating clinical trial results in critical care. Crit Care Med. 2009;37(1 Suppl):S147-53.

4. Krutsinger DC, Hetland BD, O'Leary KL, Halpern SD, Courtright KR. A Qualitative analysis of factors influencing critical care trial enrollment among surrogates. J Intensive Care Med. 2021;3:885066621998978. https://doi. org/10.1177/0885066621998978.

5. Burns KE, Zubrinich C, Tan W, Raptis S, Xiong W, Smith O, et al. Research recruitment practices and critically ill patients. A multicenter, crosssectional study (the Consent Study). Am J Respir Crit Care Med. 2013;187(11):1212-8.

6. Harhay MO, Wagner J, Ratcliffe SJ, Bronheim RS, Gopal A, Green S, et al. Outcomes and statistical power in adult critical care randomized trials. Am J Respir Crit Care Med. 2014;189(12):1469-78.

7. Paine R 3rd, Standiford TJ, Dechert RE, Moss M, Martin GS, Rosenberg $\mathrm{AL}$, et al. A randomized trial of recombinant human granulocyte-macrophage colony stimulating factor for patients with acute lung injury. Crit Care Med. 2012;40(1):90-7.

8. Chung KK, Coates EC, Smith DJ Jr, Karlnoski RA, Hickerson WL, ArnoldRoss AL, et al. High-volume hemofiltration in adult burn patients with septic shock and acute kidney injury: a multicenter randomized controlled trial. Crit Care. 2017;21(1):289. 
9. Gando S, Saitoh D, Ishikura H, Ueyama M, Otomo Y, Oda S, et al. A randomized, controlled, multicenter trial of the effects of antithrombin on disseminated intravascular coagulation in patients with sepsis. Crit Care. 2013;17(6):R297.

10. Halpern SD, Karlawish JH, Berlin JA. The continuing unethical conduct of underpowered clinical trials. JAMA. 2002;288(3):358-62.

11. Madurasinghe VW. Guidelines for reporting embedded recruitment trials. Trials. 2016;17:27

12. Harhay MO, Casey JD, Clement M, Collins SP, Gayat É, Gong MN, et al. Contemporary strategies to improve clinical trial design for critical care research: insights from the First Critical Care Clinical Trialists Workshop. Intensive Care Med. 2020:46(5):930-42.

13. Moher D, Liberati A, Tetzlaff J, Altman DG. Preferred Reporting Items for Systematic Reviews and Meta-Analyses: The PRISMA Statement. J Clin Epidemiol. 2009;62(10):1006-12

14. National Institute for Health Research PROSPERO International prospective register of systematic reviews. https://www.crd.york.ac.uk/prospero/\# aboutpage.

15. Analytics C. InCites Journal Citation Reports 2019. https://incites.clarivate. com.

16. Goldacre B, Drysdale H, Dale A, Milosevic I, Slade E, Hartley P, et al. COMPare: a prospective cohort study correcting and monitoring 58 misreported trials in real time. Trials. 2019;20(1):118.

17. Harris PA, Taylor R, Thielke R, Payne J, Gonzalez N, Conde JG. Research electronic data capture (REDCap)-a metadata-driven methodology and workflow process for providing translational research informatics support. J Biomed Inform. 2009;42(2):377-81.

18. Baker WL, White CM, Cappelleri JC, Kluger J, Coleman CI. Understanding heterogeneity in meta-analysis: the role of meta-regression. Int J Clin Pract. 2009;63(10):1426-34.

19. DerSimonian R, Laird N. Meta-analysis in clinical trials. Control Clin Trials. 1986;7(3):177-88.

20. Nalamalapu SR, Needham DM, Stapleton RD. Patient recruitment rate in multicentered randomized trials in critical care. Crit Care Med. 2016;44(7):e588-9.

21. Burns KE, Prats CJ, Maione $M$, Lanceta M, Zubrinich $C$, Jeffs $L$, et al. The experience of surrogate decision makers on being approached for consent for patient participation in research. A multicenter study. Ann Am Thorac Soc. 2017;14(2):238-45.

22. Krutsinger DC, Yadav KN, Cooney E, Brooks S, Halpern SD, Courtright KR A pilot randomized trial of five financial incentive strategies to increase study enrollment and retention rates. Contemp Clin Trials Commun. 2019;15:100390.

23. VanEpps EM, Volpp KG, Halpern SD. A nudge toward participation: improving clinical trial enrollment with behavioral economics. Sci Trans Med. 2016;8(348):348fs13.

24. Iverson E, Celious A, Kennedy CR, Shehane E, Eastman A, Warren V, et al. Real-time perspectives of surrogate decision-makers regarding critical illness research: findings of focus group participants. Chest. 2012;142(6):1433-9.

25. Lane T, Brereton E, Nowels C, McKeehan J, Moss M, Matlock DD. Surrogate informed consent: a qualitative analysis of surrogate decision makers' perspectives. Ann Am Thorac Soc. 2021;18:1185-90.

26. Tomazini BM, Maia IS, Cavalcanti AB, Berwanger $O$, Rosa RG, Veiga VC, et al. Effect of dexamethasone on days alive and ventilator-free in patients with moderate or severe acute respiratory distress syndrome and COVID-19: the CoDEX randomized clinical trial. JAMA. 2020;324(13):1307-16.

27. Grieco DL, Menga LS, Cesarano M, Rosà T, Spadaro S, Bitondo MM, et al. Effect of helmet noninvasive ventilation vs high-flow nasal oxygen on days free of respiratory support in patients with COVID-19 and moderate to severe hypoxemic respiratory failure: the HENIVOT randomized clinical trial. JAMA. 2021;325:1731-43.

28. Investigators I. Effect of intermediate-dose vs standard-dose prophylactic anticoagulation on thrombotic events, extracorporeal membrane oxygenation treatment, or mortality among patients with COVID-19 admitted to the intensive care unit: the INSPIRATION randomized clinical trial. JAMA. 2021;325(16):1620-30.

29. Sjoding MW, Prescott HC, Wunsch H, Iwashyna TJ, Cooke CR. Longitudinal changes in ICU admissions among elderly patients in the United States. Crit Care Med. 2016;44(7):1353-60.

30. Stevanovic A, Schmitz S, Rossaint R, Schürholz T, Coburn M. CONSORT item reporting quality in the top ten ranked journals of critical care medicine in 2011: a retrospective analysis. PLOS ONE. 2015;10(5):e0128061.

\section{Publisher's Note}

Springer Nature remains neutral with regard to jurisdictional claims in published maps and institutional affiliations.
Ready to submit your research? Choose BMC and benefit from:

- fast, convenient online submission

- thorough peer review by experienced researchers in your field

- rapid publication on acceptance

- support for research data, including large and complex data types

- gold Open Access which fosters wider collaboration and increased citations

- maximum visibility for your research: over $100 \mathrm{M}$ website views per year

At BMC, research is always in progress.

Learn more biomedcentral.com/submissions 